\title{
Respuestas fisiológicas y bioquímicas del fríjol caupí (Vigna unguiculata L. Walp.) bajo déficit hídrico
}

\section{Physiological and biochemical responses of the cowpea bean (Vigna unguiculata L. Walp.) under a water deficit}

CARLOS CARDONA-AYALA', 2,3

ALFREDO JARMA-OROZCO ${ }^{1}$

HERMES ARAMÉNDIZ-TATIS ${ }^{1}$

MARYORIK PEÑA-AGRESOTT ${ }^{1}$

CÉSAR VERGARA-CÓRDOBA'
Maduración de vainas de fríjol caupí tolerante a sequía.

Foto: C. Cardona-Ayala

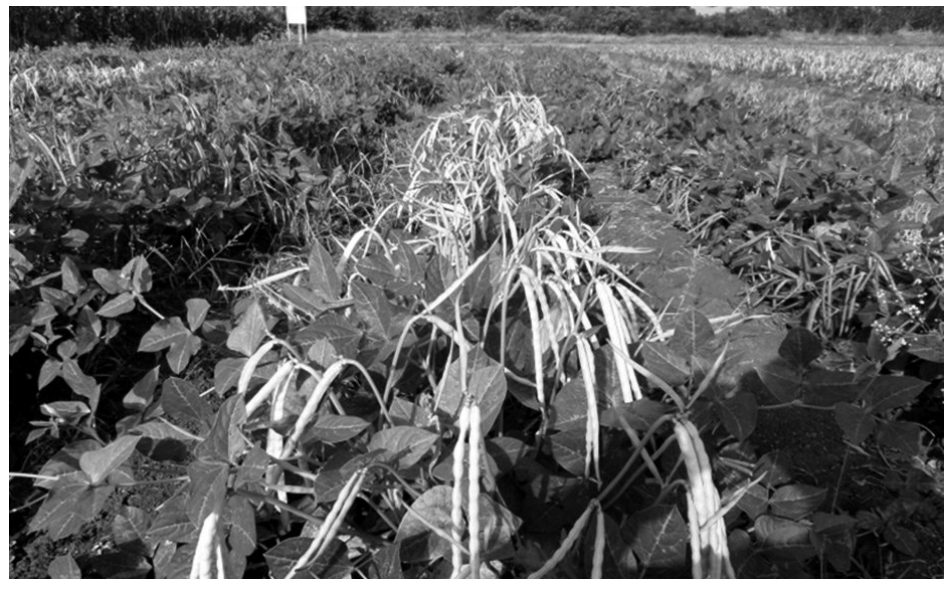

\section{RESUMEN}

El fríjol caupí contribuye a la alimentación humana y animal en muchas regiones del mundo, especialmente en aquellos lugares donde la sequía restringe la producción agrícola. El objetivo de este trabajo fue identificar algunas respuestas fisiológicas y bioquímicas de esta especie, bajo condiciones de estrés hídrico en fase reproductiva. Se experimentó bajo un diseño completamente aleatorizado en un arreglo factorial 2x6 (2 niveles de humedad del suelo y 6 genotipos) con tres repeticiones. Se analizó la respuesta de la especie evaluando el rendimiento de la planta mediante la cuantificación del rendimiento de grano/planta, el número de vainas/planta, el número de semillas/vaina, y la longitud de la vaina; se estimó la reducción relativa del rendimiento y la susceptibilidad a la sequía. También se analizó el intercambio gaseoso, la actividad de las enzimas catalasa y ascorbato peroxidasa, así como los contenidos de clorofila, carotenoides, proteínas libres, prolina y malondialdehído. El estrés por sequía causó una disminución en el rendimiento de grano/planta del 57,72\%, número de vainas/planta del 49,40\% y número de semillas/vaina del 32,07\%. A los 17 días de sequía, la fotosíntesis empezó a registrar valores cercanos a cero cuando el contenido de humedad del suelo se redujo alrededor del $40 \%$ de la capacidad de campo, lo cual pudo ser ocasionado por limitaciones estomáticas y, posiblemente, por limitaciones no estomáticas. La proporción de clorofila a/clorofila b (Chla/Chlb), fue significativamente más baja, mientras que el contenido de malondialdehído fue significativamente más alto, en plantas bajo estrés hídrico.






\section{ABSTRACT}

The cowpea bean contributes to human and animal feed in many regions of the world, especially in places where droughts restrict agricultural production. The aim of this study was to identify some physiological and biochemical responses of this species under drought stress conditions in the reproductive phase. We used a completely randomized design with a $2 \times 6$ factorial arrangement $(2$ soil moisture levels and 6 genotypes) and three replicates. The grain yield/plant, number of pods/plant, number of seeds/pod and pod length were analyzed, and the relative yield reduction and drought susceptibility index were estimated. The gas exchange and activity of the catalase and ascorbate peroxidase enzymes, as well as the contents of chlorophyll, carotenoids, free protein, and proline, were analyzed. The drought stress caused a decrease in the grain yield/ plant of $57.72 \%$, the number of pods/plant of $49.40 \%$ and the number of seeds/pod of $32.07 \%$. At 17 days of drought, the photosynthesis began showing values near zero when the soil moisture content was reduced by about $40 \%$ of the field capacity, which could have been caused by stomatal limitations and possibly by biochemical limitations. The ratio of chlorophyll a/chlorophyll $b$ (Chla/Chlb) was significantly lower and the malonaldehyde content was significantly higher in the plants under water stress.

Additional key words: crop physiology, water stress, gas exchange, antioxidant enzymes, proline.



El fríjol caupí es un cultivo importante en las regiones tropicales y subtropicales del mundo, donde la sequía es un factor de restricción en la producción debido a las bajas precipitaciones (Pungulani et al., 2013), generando pérdidas de al menos el $50 \%$ en el rendimiento de semilla (Ahmed y Suliman, 2010).

El estrés por sequía induce diversas respuestas morfofisiológicas, bioquímicas y moleculares; entre estas la fotosíntesis es afectada por la disminución de la conductancia estomática al disminuirse la difusión de $\mathrm{CO}_{2}$ desde los estomas hacia los espacios intercelulares, y luego, hacia los sitios de carboxilación a través del mesófilo, constituyendo una primera limitación (limitación estomática). Una segunda limitación, de naturaleza bioquímica conocida como limitación no estomática, está relacionada con la restricción de la fotofosforilación, la deficiente regeneración de ribulosa 1,5 -bifosfato (RuBp) y el decrecimiento en la actividad de la Rubisco (Singh y Reddy, 2011).
Ante el déficit hídrico, las limitaciones no estomáticas de la fotosíntesis se producen por la exposición de los cloroplastos a un exceso de energía de excitación, que puede ser disipada como calor vía carotenoides, fotorrespiración u otro mecanismo fotoprotector; al mismo tiempo, el exceso de energía puede generar daños en el centro de reacción del fotosistema II e incrementar la producción de especies reactivas de oxígeno (ROS, por su sigla en inglés) en los cloroplastos, tales como el oxígeno singlete, peróxido de hidrógeno, radical hidroxilo y anión superóxido, causando posibles daños en las membranas y, por tanto, aumentando el contenido de malondialdehido (MDA) (Smirnoff, 1993; Tripathy y Oelmüller, 2012).

Para contrarrestar el daño oxidativo, se activan algunos mecanismos bioquímicos como la síntesis y acumulación de osmolitos protectores y enzimas del sistema antioxidante (Tausz et al., 2004), que contribuyen al mantenimiento de los diferentes procesos fisiológicos tales como la 
apertura estomática, la fotosíntesis, la expansión celular, entre otros.

La acumulación de osmolitos compatibles es uno de los mecanismos de tolerancia a la sequía (Claeys e Inzé, 2013), el cual tiene como consecuencia la osmoprotección de las estructuras celulares de las plantas ante el déficit hídrico y está dada por la capacidad estabilizadora de algunos de estos solutos sobre macromoléculas como las proteínas y los sistemas de membrana celulares. Moléculas como prolina y glicina-betaína, pueden inducir la formación de puentes de hidrógeno alrededor de proteínas, preservando su estado original (Taiz y Zeiger, 2010).

Entre las enzimas del sistema antioxidante involucradas en procesos de detoxificación de ROS están la catalasa (CAT), ascorbato peroxidasa (APX), superóxido dismutasa (SOD) y glutatión reductasa (GR) (Pompelli et al., 2010). Las enzimas GR y APX desempeñan un papel clave en fríjol, debido a que la regulación de la expresión del gen de la GR citosólica (cGR) está directamente relacionada con la intensidad del estrés hídrico (Agbicodo et al., 2009).

Al considerar que el frijol caupí se presenta como una alternativa en zonas donde se presentan periodos de sequía prolongados como en el Sinú Medio colombiano, el estudio tuvo como objetivo evaluar algunas respuestas fisiológicas y bioquímicas de seis genotipos de esta especie sometidos a déficit hídrico progresivo.

\section{MATERIALES Y MÉTODOS}

\section{Material vegetal y condiciones experimentales}

El experimento se realizó en un invernadero cubierto con plástico en la estación experimental de la Universidad de Córdoba (848' N, 7853' W) localizada en Montería, Colombia, entre mayo y julio de 2013. Semillas de las líneas promisorias del Programa de Mejoramiento
Genético en Fríjol Caupí de la Universidad de Córdoba, L-001, L-006, L-034, L-047 y de los testigos Criollo-Córdoba (parental de las líneas) y BRS-Milenium, fueron sembradas en bolsas plásticas con $24,5 \mathrm{~kg}$ de suelo franco-limoso a capacidad de campo. Se utilizó un diseño experimental completamente aleatorizado con arreglo factorial $6 \times 2$ y tres repeticiones. El primer factor correspondió al genotipo y el segundo a humedad del suelo (con riego y sin riego, a partir de floración).

El contenido gravimétrico de agua (p/p) fue determinado cada $4 \mathrm{~d}$, a la profundidad de $20 \mathrm{~cm}$. Las muestras de suelo (tres/unidad experimental), de aproximadamente $200 \mathrm{~g}$, se tomaron de contenedores (bolsas plásticas) con plantas destinadas para muestreo destructivo. Los recipientes con el material húmedo se introdujeron en un horno de secado a $110 \pm 5^{\circ} \mathrm{C}$, por $24 \mathrm{~h}$ (Forsythe, 1985). También se determinó el contenido volumétrico de agua $(\mathrm{v} / \mathrm{v})$ con el medidor TDR 300 (Spectrum Technologies, Plainfield, IL), a 20 $\mathrm{cm}$ de profundidad en todos los recipientes.

\section{Caracteres morfofisiológicos}

A los 29 d de haber suspendido el suministro de agua, se determinó el rendimiento de grano/ planta (RGP), el número de vainas/planta (NVP), el número de semillas/vaina (NSV), la longitud de la vaina (LV), y los siguientes índices:

Reducción relativa del rendimiento RRR: se restó el rendimiento de grano en sequía al rendimiento de grano en riego y se dividió por el rendimiento de grano en riego.

Índice de intensidad de sequía (IIS): se estimó mediante la fórmula:

$$
\mathrm{IIS}=1-(\mathrm{Ys} / \mathrm{Yr})
$$

Donde Ys y Yr son las medias de rendimiento de todos los genotipos bajo tratamientos de sequía (s) y riego (r), respectivamente. 
Índice de susceptibilidad sequía (ISS): se calculó mediante la fórmula:

$$
\mathrm{ISS}=\left[\left(1-\left(\mathrm{Ys}_{\mathrm{i}} / \mathrm{Yr}_{\mathrm{i}}\right)\right] / \mathrm{IIS}\right.
$$

Donde $\mathrm{Ys}_{\mathrm{i}} \mathrm{y} \mathrm{Yr}_{\mathrm{i}}$ son las medias de rendimiento de cada genotipo en condiciones de sequía y riego respectivamente (Fisher y Maurer, 1978).

\section{Medidas de intercambio gaseoso}

Se determinaron las tasas de fotosíntesis $A$ $\left(\mu \mathrm{mol} \mathrm{m}^{-2} \mathrm{~s}^{-1}\right)$, conductancia estomática $g_{\mathrm{s}}$ $\left(\mathrm{mmol} \mathrm{m} \mathrm{s}^{-2} \mathrm{~s}^{-1}\right)$, transpiración $E\left(\mathrm{mmol} \mathrm{m} \mathrm{m}^{-2} \mathrm{~s}^{-1}\right)$, eficiencia intrínseca en el uso del agua $A / g_{s}$, $A / E$, y $\mathrm{CO}_{2}$ intercelular y ambiental $(\mu \mathrm{mol} \mathrm{mol}$ ${ }^{1}$ ) cada $4 \mathrm{~d}$, durante $28 \mathrm{~d}$; para ello se usó un analizador de gases en el infrarrojo (IRGA), medidor portátil de fotosíntesis modelo CIRAS-2 (PP Systems International, Amesbury, MA). Las medidas se hicieron entre las 8 y las $11 \mathrm{~h}$ sobre un foliolo de la segunda, tercera o cuarta hoja sana y totalmente expandida, contadas desde el ápice de la planta.

La temperatura media del aire fue de $31,44 \pm$ $7,76^{\circ} \mathrm{C}$; la media máxima de $45,62 \pm 2,73^{\circ} \mathrm{C}$, registrada entre las 9:00 a.m. y 15:00 p.m. y la media mínima de $25,12 \pm 0,76^{\circ} \mathrm{C}$, registrada entre las 04:00 a.m. y 06:00 a.m. La temperatura máxima registrada fue de $51,1^{\circ} \mathrm{C}$ y la mínima de $22,9^{\circ} \mathrm{C}$. La humedad relativa media fue de 70,54 $\pm 22,92 \%$, con valores que fluctuaron entre $22 \mathrm{y}$ $70 \%$ durante el día y entre 80 y $97 \%$ durante la noche. Estas medidas fueron tomadas automáticamente con el registrador gráfico de humedad y temperatura, modelo RH520 (Extech Instruments, Nashua, $\mathrm{NH}$ ).

\section{Determinaciones analíticas}

\section{Muestras}

Para las determinaciones analíticas, se tomaron 108 muestras de hojas totalmente expandidas de la parte apical de las plantas (tercera o cuarta hoja) distribuidas en tres grupos de 36 (12 tratamientos $\mathrm{x} 3$ repeticiones). Un grupo para determinación de clorofilas, carotenoides y MDA; el segundo grupo para proteínas libres y prolina $y$, el tercero, para las enzimas CAT y APX. Las muestras se tomaron a los $17 \mathrm{~d}$ posteriores al inicio de la floración, cuando las plantas estresadas presentaron valores de fotosíntesis y conductancia estomática cercanos a cero, y se conservaron en nitrógeno líquido hasta su uso.

\section{Extracción y actividad de catalasa (CAT) y ascorbato peroxidasa (APX)}

La extracción de enzimas del tejido foliar (100 mg), se hizo mediante molido en frío con mortero de porcelana en baño de hielo hasta que el tejido se visualizó sin fibras. Se utilizaron $50 \mathrm{~mL}$ de solución tampón que constituyó el medio de extracción constituido por tampón fosfato de potasio (TFK) $100 \mathrm{mM}$ (pH 6,5), ácido etilendiaminotetraacético (EDTA) $2 \mathrm{mM}$, ascorbato 20 $\mathrm{mM}$ y polivinilpolipirrolidona (PVPP) $30 \% \mathrm{p} / \mathrm{p}$. La homogenización se hizo mediante centrifugado a 15.000 x g por 15 min en una centrífuga refrigerada a $4^{\circ} \mathrm{C}$. El sobrenadante se utilizó para la determinación de la actividad de las enzimas CAT y APX.

Las actividades de CAT y APX se midieron en términos de la descomposición de $\mathrm{H}_{2} \mathrm{O}_{2}$ y ascorbato, respectivamente. La descomposición del $\mathrm{H}_{2} \mathrm{O}_{2}$ por la CAT se determinó por la medida de la tasa de disminución de la absorbancia a 240 $\mathrm{nm}$ por $1 \mathrm{~min}$, en una solución de $12,5 \mathrm{mM}$ de $\mathrm{H}_{2} \mathrm{O}_{2}$ en $50 \mathrm{mM}$ del tampón K-fosfato (TFK $\mathrm{pH} 7,0)$ a $30^{\circ} \mathrm{C}$. Un $\mathrm{mL}$ de la mezcla de reacción contenía $960 \mu \mathrm{L}$ del tampón TFK (pH 7,0), 25 $\mu \mathrm{L}$ de $\mathrm{H}_{2} \mathrm{O}_{2}$ y $15 \mu \mathrm{L}$ del extracto de la enzima (Havir y McHale, 1987). La descomposición de $\mathrm{H}_{2} \mathrm{O}_{2}$ por la APX se midió por la disminución de la absorbancia a $290 \mathrm{~nm}$ durante $1 \mathrm{~min}$, en una solución que contenía $0,1 \mathrm{mM}$ de $\mathrm{H}_{2} \mathrm{O}_{2}$ en 50 mM del tampón K-fosfato (TFK - pH 6,0) y $0,5 \mathrm{mM}$ de ácido ascórbico a $25^{\circ} \mathrm{C}$. Un $\mathrm{mL}$ de la mezcla de reacción contenía $925 \mu \mathrm{L}$ del tam- 
pón TFK (pH 6,0), $10 \mu \mathrm{L}$ de $\mathrm{H}_{2} \mathrm{O}_{2}, 50 \mu \mathrm{L}$ de ácido ascórbico y $15 \mu \mathrm{L}$ del extracto de la enzima (Nakano y Asada, 1981). Las reacciones se iniciaron con el extracto de las enzimas. Una unidad de CAT o APX se definió como la cantidad de enzima requerida para oxidar $1 \mu \mathrm{M}$ de $\mathrm{H}_{2} \mathrm{O}_{2} \mathrm{O}$ ascorbato por minuto.

Determinación de malondialdehído (MDA), clorofilas, carotenoides, prolina y proteínas libres

Para la determinación de MDA, las muestras (50 mg de tejido foliar) se homogenizaron en $2 \mathrm{~mL}$ de ácido tricloroacético TCA de $0,1 \%$ (v/v) y se centrifugaron a $10.000 \mathrm{xg}$ por $15 \mathrm{~min}$. El MDA de los extractos se determinó mediante la reacción del ácido tiobarbitúrico (TBA), según el procedimiento de Cakmak y Horst (1991).

Los contenidos de clorofilas y carotenoides (a partir de $100 \mathrm{~g}$ de tejido foliar) se determinaron espectrofotométricamente en el extracto según la metodología de Lichtenthaler (1987); proteínas libres (50 mg de tejido foliar), según Bradford (1976) y prolina (100 mg de tejido foliar), según Bates (1973).

\section{Análisis estadísticos}

Se efectuaron análisis de varianza y pruebas de comparación de medias de Tukey al 5\%, con el software SAS versión 9.2 (SAS, 2008).

\section{RESULTADOS Y DISCUSIÓN}

\section{Caracteres morfofisiológicos}

La suspensión del agua a partir de floración redujo la totalidad de los caracteres evaluados. E1 RGP y el NSV mostraron efectos significativos para la interacción genotipo por humedad, indicando que existe un comportamiento diferencial de los genotipos ante la presión del estrés; por su parte, el NVP y la LV mostraron diferencias $(P \leq 0,01)$ solo para los efectos principales del genotipo y el nivel de humedad del suelo (tabla 1, figura 1). Resultados similares fueron reportados por Ahmed y Suliman (2010), quienes atribuyen la disminución del rendimiento y sus componentes, bajo sequía, a la abscisión de estructuras reproductivas y la limitación de fotoasimilados para la formación y llenado de granos (Ishiyaku y Aliyu, 2013). En varios estudios se ha reiterado el efecto deletéreo de la sequía sobre el crecimiento, el rendimiento y la nutrición mineral de las plantas (Afshar et al., 2013).

El estrés por sequía causó disminución del rendimiento de grano por planta de $57,72 \%$, del número de vainas por planta de $49,40 \%$, del número de semillas por vaina de $32,07 \%$ y de la longitud de la vaina de 13,93\%, con una correlación altamente significativa entre los dos primeros caracteres mencionados, lo que posi-

Tabla 1. Cuadrados medios del análisis de varianza de seis genotipos de caupí bajo déficit de humedad del suelo para el rendimiento de grano por planta (RGP), número de vainas por planta (NVP), número de semillas por vaina (NSV) y longitud de la vaina (LV).

\begin{tabular}{|l|c|c|c|c|}
\multicolumn{1}{r|}{ FV } & RGP & NVP & NSV & LV \\
\hline Humedad & $104,05^{* *}$ & $38,05^{* *}$ & $62,23^{* *}$ & $45^{* *}$ \\
\hline Genotipos & $5,66^{* *}$ & $2,56^{* *}$ & $19,89^{* *}$ & $0,86 \mathrm{~ns}$ \\
\hline Interacción & $3,22^{* *}$ & $0,36 \mathrm{~ns}$ & $3,56^{*}$ & 12,82 \\
\hline Media & 4,19 & 3,14 & 6,89 & 7,19 \\
\hline CV (\%) & 16,41 & 12,52 & 15,38 & \\
\hline
\end{tabular}

${ }^{*}=$ significativa $(P \leq 0,05) ;{ }^{* *}=$ altamente significativa $(P \leq 0,01) ; n s=$ no significativa; $C V(\%)=$ porcentaje del coeficiente de variación. 




Figura 1. Rendimiento de grano/planta de los genotipos de fríjol caupí según el nivel de humedad del suelo (riego vs. sequía).

blemente obedezca a la limitada actividad fotosintética y a una menor absorción de nutrientes por la reducida movilidad de iones en el suelo y toma de agua por las raíces. Ahmed y Suliman (2010), en trabajos desarrollados en campo, reportaron disminución del rendimiento de semilla/ha entre el 50 y $74 \%$ en esta especie.
Los resultados evidenciaron variabilidad entre genotipos en cuanto a la reducción del rendimiento, tal como lo reportan otros trabajos (Abayomi y Abidoye, 2009; Ishiyaku y Aliyu, 2013; Cardona et al., 2013); algunos genotipos presentaron mayor reducción relativa del rendimiento, consistente con un menor índice de susceptibilidad a sequía (tabla 2). Varios estudios han indicado

Tabla 2. Valores medios ( \pm error estándar) del rendimiento de grano/planta (g) (RP); reducción relativa del rendimiento (RRR) e índice de susceptibilidad a sequía (ISS) en seis genotipos de caupí.

\begin{tabular}{|c|c|c|c|c|}
\hline \multirow{2}{*}{ Genotipo } & \multicolumn{2}{|c|}{$\mathrm{RP}$} & \multirow{2}{*}{$\begin{array}{c}\text { RRR } \\
\text { Riego-sequía }\end{array}$} & \multirow{2}{*}{$\begin{array}{c}\text { ISS } \\
\text { Riego-sequía }\end{array}$} \\
\hline & Riego & Sequía & & \\
\hline L-001 & $3,00 \pm 0,63 b$ & $1,74 \pm 0,07 c$ & $0,38 \pm 0,09 a$ & $0,67 \pm 0,16 a$ \\
\hline L-006 & $6,97 \pm 0,27 a$ & $2,73 \pm 0,27 a b$ & $0,61 \pm 0,03 a b$ & $1,07 \pm 0,05 a b$ \\
\hline L-034 & $7,10 \pm 0,22 \mathrm{a}$ & $2,57 \pm 0,21 b c$ & $0,64 \pm 0,03 b$ & $1,12 \pm 0,05 b$ \\
\hline L-047 & $5,39 \pm 0,57 a b$ & $2,25 \pm 0,19 b c$ & $0,58 \pm 0,01 a b$ & $1,03 \pm 0,02 a b$ \\
\hline BRS-MIL & $7,18 \pm 0,55 a$ & $2,15 \pm 0,17 \mathrm{bc}$ & $0,69 \pm 0,05 b$ & $1,22 \pm 0,08 b$ \\
\hline CR-CO & $5,71 \pm 0,74 a$ & $3,52 \pm 0,18 a$ & $0,37 \pm 0,06 a$ & $0,65 \pm 0,11 \mathrm{a}$ \\
\hline Media & $5,89 \pm 0,22$ & $2,49 \pm 0,33$ & $0,54 \pm 0,09$ & $0,96 \pm 0,16$ \\
\hline
\end{tabular}

Promedios con letras distintas indican diferencia significativa según la prueba de Tukey $(P \leq 0,05)$. 
que el ISS podría considerarse como un criterio aceptable para discriminar genotipos; sin embargo debe tomarse con precaución y relacionarse con otras características, ya que las líneas con mayor tolerancia a sequía, no necesariamente son las más rendidoras bajo esta condición, pero sí las que menos reducen su rendimiento al variar la oferta hídrica (Rosales-Serna et al., 2000). Bajo este argumento, la línea L-001 y el parental CR-CO se mostraron como los más tolerantes, seguidos por L-047 y L-006.

\section{Intercambio gaseoso}

El efecto de la disminución del contenido de humedad del suelo se hizo evidente a partir de los $5 \mathrm{~d}$ posteriores a la suspensión del suministro de agua a las plantas, con disminuciones altamente significativas $(P \leq 0,01)$ de $A, g_{s} y E$ (tabla 3$)$.

A los $17 \mathrm{~d}$, cuando la humedad del suelo se había reducido cerca al 40\% de la humedad a capacidad de campo, $A, g_{\mathrm{s}}$ y $E$ junto a $A / g_{\mathrm{s}} \mathrm{y} A / E$

Tabla 3. Valores medios ( \pm error estándar) de fotosíntesis neta $(A)$, conductancia estomática $\left(g_{s}\right)$, transpiración $(E)$, eficiencia intrínseca en el uso del agua $\left(A / g_{s}\right)$, eficiencia de transpiración $(A / E)$ y proporción entre el $\mathrm{CO}_{2}$ intercelular y el $\mathrm{CO}_{2}$ ambiental $\left(C_{*} / C_{\mathrm{a}}\right)$ en hojas de fríjol caupí durante estrés hídrico a partir de floración. Estimaciones seguidas por * ***a fueron significantes frente al control a los niveles de $5 \%$ y $1 \%$, respectivamente.

\begin{tabular}{|c|c|c|c|c|c|c|c|}
\hline \multirow{2}{*}{$\begin{array}{l}\text { Tiempo } \\
\text { (d) }\end{array}$} & \multirow{2}{*}{$\begin{array}{c}\text { Humedad } \\
(\% \mathrm{p} / \mathrm{p})\end{array}$} & \multicolumn{2}{|c|}{$A\left(\mu \mathrm{mol} \mathrm{CO}_{2} \mathrm{~m}^{-2} \mathrm{~s}^{-1}\right)$} & \multicolumn{2}{|c|}{$g_{s}\left(\mathrm{~mol} \mathrm{H} \mathbf{O} \mathrm{m}^{-2} \mathrm{~s}^{-1}\right)$} & \multicolumn{2}{|c|}{$E\left(m m o l ~ H_{2} O m^{-2} s^{-1}\right)$} \\
\hline & & Riego & Sequía & Riego & Sequía & Riego & Sequía \\
\hline 1 & 26,66 & $18,75 \pm 0,57$ & $17,29 \pm 0,57$ & $76,03 \pm 3,86$ & $53,73 \pm 3,86^{* *}$ & $2,48 \pm 0,14$ & $2,07 \pm 0,14$ \\
\hline 5 & 25,91 & $13,41 \pm 0,34$ & $3,23 \pm 0,34^{* *}$ & $62,68 \pm 3,52$ & $34,17 \pm 3,52^{* *}$ & $2.16 \pm 0,10$ & $0.93 \pm 0,10^{* *}$ \\
\hline 9 & 23,84 & $9,07 \pm 0,18$ & $2,21 \pm 0,18^{* *}$ & $41,61 \pm 0,94$ & $26,19 \pm 0,94^{* *}$ & $1,59 \pm 0,03$ & $0,83 \pm 0,03^{* *}$ \\
\hline 13 & 21,66 & $8,75 \pm 0,29$ & $2,25 \pm 0,29 * *$ & $26,61 \pm 0,85$ & $12,69 \pm 0,85^{* *}$ & $1,13 \pm 0,05$ & $0,60 \pm 0,05^{* *}$ \\
\hline 17 & 19,54 & $10,81 \pm 0,29$ & $-0,94 \pm 0,29^{* *}$ & $50,33 \pm 1,74$ & $11,54 \pm 1,74^{* *}$ & $1,57 \pm 0,05$ & $0,47 \pm 0,05^{* *}$ \\
\hline 21 & 18,40 & $10,51 \pm 0,35$ & $-1,71 \pm 0,35^{* *}$ & $77,62 \pm 3,66$ & $6,07 \pm 3,66^{* *}$ & $4,02 \pm 0,05$ & $0,28 \pm 0,05^{* *}$ \\
\hline 25 & 15,85 & $12,74 \pm 0,23$ & $-0,39 \pm 0,23^{* *}$ & $48,50 \pm 1,38$ & $8,28 \pm 1,38^{* *}$ & $1,46 \pm 0,03$ & $0,33 \pm 0,03^{* *}$ \\
\hline 29 & 14,99 & $11,42 \pm 0,27$ & $-2,32 \pm 0,27^{* *}$ & $59,19 \pm 1,17$ & $6,22 \pm 1,17^{* *}$ & $1,61 \pm 0,04$ & $0,23 \pm 0,04^{* *}$ \\
\hline \multirow{2}{*}{$\begin{array}{c}\text { Tiempo } \\
\text { (d) }\end{array}$} & \multirow{2}{*}{$\begin{array}{c}\text { Humedad } \\
(\% p / p)\end{array}$} & \multicolumn{2}{|c|}{$A / g_{\mathrm{s}}\left(\mu \mathrm{mol} \mathrm{CO} \mathrm{mol}^{-1} \mathrm{H}_{2} \mathrm{O}\right)$} & \multicolumn{2}{|c|}{$A / E\left(\mu \mathrm{mol} \mathrm{CO} \mathrm{mmol}^{-1} \mathrm{H}_{2} \mathrm{O}\right)$} & \multicolumn{2}{|c|}{$C_{i} / C_{a}$} \\
\hline & & Riego & Sequía & Riego & Sequía & Riego & Sequía \\
\hline 1 & 26,66 & $0,36 \pm 0,02^{*}$ & $0,28 \pm 0,02$ & $17,29 \pm 0,57^{*}$ & $18,75 \pm 0,57$ & $0,013 \pm 0,01^{*}$ & $0,06 \pm 0,01$ \\
\hline 5 & 25,91 & $0,10 \pm 0.05^{*}$ & $0,31 \pm 0.05$ & $3,23 \pm 0,34^{* *}$ & $13,41 \pm 0,34$ & $0,16 \pm 0,02^{*}$ & $0,05 \pm 0,02$ \\
\hline 9 & 23,84 & $0,08 \pm 0,01^{* *}$ & $0,23 \pm 0,01$ & $2,21 \pm 0,18^{* *}$ & $9,07 \pm 0,18$ & $0,57 \pm 0,06^{* *}$ & $0,08 \pm 0,06$ \\
\hline 13 & 21,66 & $0,17 \pm 0,0^{* *}$ & $0,34 \pm 0,02$ & $3,79 \pm 0,35^{* *}$ & $8,07 \pm 0,35$ & $0,81 \pm 0,03^{* *}$ & $0,02 \pm 0,03$ \\
\hline 17 & 19,54 & $-0,08 \pm 0,02^{* *}$ & $0,22 \pm 0,02$ & $-2,14 \pm 0,46^{* *}$ & $7,12 \pm 0,46$ & $0,73 \pm 0,08^{*}$ & $0,10 \pm 0,08$ \\
\hline 21 & 18,40 & $-0,38 \pm 0,05^{* *}$ & $0,15 \pm 0,05$ & $-9,67 \pm 1,00^{* *}$ & $2,60 \pm 1,00$ & $1,03 \pm 0,06^{* *}$ & $0,17 \pm 0,06$ \\
\hline 25 & 15,85 & $-0,07 \pm 0,01^{* *}$ & $0,27 \pm 0,01$ & $-1,73 \pm 0,30^{* *}$ & $8,85 \pm 0,30$ & $3,18 \pm 0,18^{*}$ & $0,10 \pm 0,18$ \\
\hline 29 & 14,99 & $0.20 \pm 0.07^{* *}$ & $-0.38 \pm 0.07$ & $7.26 \pm 2.09^{* *}$ & $-10.39 \pm 2.09$ & $1,00 \pm 0,02^{* *}$ & $0,14 \pm 0,02$ \\
\hline
\end{tabular}


alcanzaron promedios muy bajos, a tal punto que la fotosíntesis empezó a registrar valores negativos, debido posiblemente al $\mathrm{CO}_{2}$ producto de la respiración, con balance negativo para fotosíntesis neta. Sin embargo, no se observaron diferencias significativas entre genotipos, lo que posiblemente obedezca a una tolerancia similar a la sequía, identificada en un estudio anterior con los mismos materiales (Cardona et al., 2013).

La disminución de $A$ se atribuye a una limitación cuantitativa de la misma por efecto de las limitaciones estomáticas y posiblemente no estomáticas que aumentan con la sequía, con un mayor efecto de la segunda cuando el estrés hídrico es severo. Es posible que en tales condiciones y con temperaturas elevadas, pudiera haberse impedido el transporte de electrones o verse disminuida la actividad de la rubisco, dado que en determinadas horas del día se registraron temperaturas muy altas que hubiesen podido superar valores críticos para la estabilidad del fotosistema II y la capacidad de carboxilación (Grassi y Magnani, 2005). En tales condiciones también es posible la inactivación de las enzimas del ciclo de Calvin, bien por falta de ATP o por deficiente síntesis de RUBP (Lawlor, 2002).

La disminución de $A / E$ a nivel de la hoja no mostró diferencias entre genotipos, posiblemente por la similitud de los mismos en relación con tolerancia a sequía y por la variabilidad intrínseca de esta respuesta. Sin embargo, Blum (2009) ha reportado que $A / E$, a nivel de la hoja, es más alta en genotipos tolerantes a sequía en condiciones de estrés hídrico y también puede ser medida por discriminación isotópica de carbono $\left(\delta^{13} \mathrm{C}\right)$ en granos. Un trabajo similar reportó que en condiciones de estrés hídrico severo, varias líneas de caupí tolerantes a sequía, mostraron conductancia estomática y eficiencia de transpiración más altas que las sensibles (Belko et al., 2012).

Por otro lado, para el mismo periodo de observación, la proporción entre el $\mathrm{CO}_{2}$ intercelular y el $\mathrm{CO}_{2}$ ambiental $\left(C_{\mathrm{i}} / C_{\mathrm{a}}\right)$ (tabla 3 ) resultó inversamente proporcional a $A, g_{\mathrm{s}}, A / g_{\mathrm{s}}$ y $A / E$ y, en la medida en que el estrés hídrico se hizo más intenso, $C_{i} / C_{a}$ también aumentó notablemente. Al respecto, Brodribb (1996) reportó que cuando el estrés hídrico se hace más fuerte, esta proporción aumenta como consecuencia de la limitación no estomática de la fotosíntesis. Singh y Reddy (2011) encontraron $C_{\mathrm{i}} / C_{\mathrm{a}}$ inversamente proporcional a $A / g_{s}$ en caupí y, Martin y RuizTorres (1992), en trigo. La magnitud de $A$ estimada con métodos de regresión, alcanzó el valor de cero cuando $C_{\mathrm{i}}$ alcanzó $250 \mu \mathrm{mol} \mathrm{mol}{ }^{-1}$, 38\% más alto que el estimado por Singh y Reddy (2011) en caupí, en un estudio similar.

\section{Actividad bioquímica}

Los contenidos de clorofilas a y b, no evidenciaron disminución significativa durante estrés hídrico, como ha ocurrido en otros estudios (Pompelli et al., 2010; Witta et al., 2012), pero Chla/Chlb fue significativamente más baja en plantas estresadas.

La reducción de clorofilas inducida por la sequía ha sido reportada en numerosos estudios y constituye un mecanismo de defensa de la planta para reducir la presión del exceso de energía sobre los fotosistemas I y II (Iturbe-Ormaetxe et al., 1998; Pompelli et al., 2010). En este estudio la disminución de clorofila a, fue de $8,4 \%$, sin diferencias significativas entre cultivares, cifra mucho menor que la reportada por Bastos et al. (2011) en esta misma especie, de 19\%, quienes además indicaron que aquellos cultivares que registraron menor reducción de clorofila, tuvieron mejor rendimiento de grano.

Aunque los niveles de carotenoides no aumentaron significativamente durante sequía, la disminución significativa de Chla/Chlb, podría sugerir una fotoprotección efectiva (Pompelli et al., 2010) ya que la clorofila $b$ y los carotenoides absorben luz de longitudes diferentes de las que absorbe la clorofila a y actúan como pantallas que transfieren la energía a la clorofila $a$, ex- 
tendiendo así la gama de luz disponible para la fotosíntesis, pero al mismo tiempo la energía de excitación excesiva es reorientada y disipada principalmente como calor, para lograr un balance entre la energía recibida por el fotosistema II y la capacidad de este para utilizarla (Cabrera, 2002).

El contenido de prolina aumentó 8,3\% en condición de sequía, inferior al encontrado por Costa et al. (2008) en esta especie (45,3\%), por lo que no hubo deferencia significativa con el control (tabla 4). Al respecto, Santos et al. (2010) encontraron aumentos significativos de prolina en genotipos de fríjol caupí intermedios, pero en genotipos tardíos no se evidenció tal aumento.

Por otro lado, el contenido de proteínas no varió significativamente bajo sequía, lo que podría estar relacionado con la tolerancia a sequía de los genotipos estudiados, ya que la disminución de proteínas y la salida de solutos de las células son indicadores de senescencia en tejidos verdes (Beltrano et al., 2006).
La actividad de CAT registró un aumento de 33\% en plantas estresadas, significativo al 10\%, con respecto a plantas irrigadas (tabla 4). Sin embargo, varios estudios reportan cambios ligeros solamente en la actividad de esta enzima localizada en los peroxisomas (Smirnoff, 1993). Por otro lado, la actividad de APX registró aumento de $13,5 \%$ en plantas estresadas, sin diferencias significativas con respecto a plantas irrigadas. Varios estudios reportan que la adaptación a sequía se manifiesta en la capacidad de incrementar los niveles de esta enzima antioxidante (Pompelli et al., 2011). Sin embargo, se ha reportado que durante fuerte estrés oxidativo, resultante de la sequía en esta especie, se podría reducir la actividad de APX posiblemente por disminución del antioxidante ascorbato (Iturbe-Ormaetxe et al., 1998), lo que pudo haber ocurrido en este estudio.

Concomitante con lo anterior, Santos et al. (2010) encontraron que el estrés hídrico induce una respuesta diferencial en la actividad de las peroxidasas resultando mayor o menor de acuerdo con el genotipo de caupí estudiado (Fernan-

Tabla 4. Valores medios ( \pm error estándar) de clorofilas, carotenoides, proteínas, prolina, enzimas y aldehído malónico en hojas de caupí durante estrés hídrico a partir de floración.

\begin{tabular}{|c|c|c|c|c|}
\hline Biomoléculas & Sequía & Riego & \multicolumn{2}{|c|}{ Diferencia } \\
\hline Clorofila a (g kg-1 MS) & $7,35 \pm 0,18$ & $8,02 \pm 0,18$ & $-0,67$ & * \\
\hline Clorofila $b$ (g kg-1 MS) & $4,11 \pm 0,10$ & $4,25 \pm 0,10$ & $-0,14$ & ns \\
\hline Chlorofila a /Clorofila b (Chla/Chlab) & $1,79 \pm 0,02$ & $1,90 \pm 0,02$ & $-0,11$ & ** \\
\hline Carotenoides ( $\left.\mathrm{g} \mathrm{kg}^{-1} \mathrm{MS}\right)$ & $1,39 \pm 0,03$ & $1,38 \pm 0,03$ & 0,01 & ns \\
\hline Clorofila/Carotenoides & $8,51 \pm 0,25$ & $9,04 \pm 0,25$ & $-0,52$ & $\mathrm{~ns}$ \\
\hline Proteínas solubles ( $\mathrm{g} \mathrm{kg}^{-1} \mathrm{MS}$ ) & $292,72 \pm 14,51$ & $287,48 \pm 14,51$ & 5,24 & ns \\
\hline Prolina ( $\left.\mu \mathrm{M} \mathrm{g}^{-1} \mathrm{MS}\right)$ & $0,182 \pm 0,02$ & $0,168 \pm 0,02$ & 0,014 & ns \\
\hline Catalasa (U/mg proteína) & $0,12 \pm 0,02$ & $0,08 \pm 0,02$ & 0,04 & * \\
\hline $\begin{array}{l}\text { Ascorbato peroxidasa } \\
\text { (U/mg proteína) }\end{array}$ & $0,42 \pm 0,07$ & $0,37 \pm 0,07$ & 0,05 & ns \\
\hline Aldehido malónico ( $\left.\mu \mathrm{M} \mathrm{kg}^{-1} \mathrm{MS}\right)$ & $163,82 \pm 7,89$ & $126,72 \pm 7,89$ & 37.1 & ** \\
\hline
\end{tabular}

${ }^{*}=$ significativa al $10 \% ;{ }^{* *}=$ significativa al $5 \% ;$ ns = no significativa; $M S=$ materia seca. 
des-Santos et al., 2010), variación que se pueden explicar de acuerdo con la adaptación que ha tenido cada material a tal condición.

El contenido de MDA, uno de los últimos productos de la peroxidación de lípidos (Pompelli et al., 2011), alcanzó un valor significativamente más alto en plantas estresadas, lo que sugiere que a los $17 \mathrm{~d}$ después de iniciada la suspensión del suministro de agua, se evidenciaban posibles daños de las membranas celulares por efecto de los radicales libres, sin diferencias importantes entre los genotipos estudiados, corroborando lo antes anotado en cuanto a la respuesta similar de los genotipos a la sequía.

\section{CONCLUSIONES}

El estrés hídrico indujo disminuciones significativas en el intercambio gaseoso, especialmente en conductancia estomática al disminuir la actividad fotosintética en caupí, lo que trajo como consecuencia una menor producción de biomasa. Así, el rendimiento de grano/planta y el número de semillas/vaina varió según el nivel de humedad del suelo (riego vs. sequía), pero estos caracteres disminuyeron significativamente durante sequía (mayor reducción relativa del rendimiento e índice de susceptibilidad a sequía). Esta variación permitió identificar los genotipos CR-CO, L-001, L-006 y L-047, como los más tolerantes. No obstante, L-001 presentó menor rendimiento de grano/planta en ambas condiciones de humedad del suelo.

El contenido de clorofila a disminuyó durante sequía, mientras que el de Chla/Chlb aumentó, lo que sugiere una fotoprotección efectiva por la acción conjunta de clorofila $b$ y carotenoides. La actividad de CAT aumentó significativamente durante estrés hídrico, mientras que APX tuvo un aumento leve, posiblemente por la disminución del ascorbato, a raíz del fuerte estrés oxidativo resultante del largo periodo de sequía. El contenido de proteínas libres no varió, lo que podría estar relacionado con la tolerancia a sequía de los genotipos estudiados, mientras que el contenido de prolina aumentó ligeramente. El incremento significativo de MDA evidenció posibles daños de las membranas celulares por efecto de los radicales libres, sin diferencias importantes entre los genotipos estudiados, corroborando lo antes anotado en relación con una respuesta similar de los genotipos a la sequía.

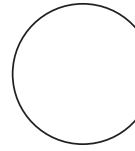

Abayomi, Y.A. y T.O. Abidoye. 2009. Evaluation of cowpea genotypes for soil moisture stress tolerance under screen house conditions. Afr. J. Plant Sci. 3(10), 229-237.

Afshar, R.M., H. Hadi y A. Pirzard. 2013. Effect of nanoiron on the yield and yield component of cowpea (Vigna unguiculata) under end season water deficit. Int. J. Agric. Res. Rev. 3(1), 27-34.

Agbicodo, E.M., C.A. Fatokun, S. Muranaka, R.G.F Visser y C.G. Linden Van Der. 2009. Breeding drought tolerant cowpea: constraints, accomplishments, and future prospects. Euphytica 167, 353-370. Doi: 10.1007/s10681-009-9893-8

Ahmed, F.E. y A.S.H. Suliman. 2010. Effect of water stress applied at different stages of growth on seed

\section{REFERENCIAS BIBLIOGRÁFICAS}

yield and water-use efficiency of cowpea. Agric. Biol. J. North America 1(4), 534-540.

Bastos, E.A., S. Nascimento, E. Silva, F. Freire y R. Gomide. 2011. Identification of cowpea genotypes for drought tolerance. Rev. Ciênc. Agron. 42(1), 100 107. Doi: 10.1590/S1806-66902011000100013

Bates, L.S. 1973. Rapid determination of free proline for water stress studies. Plant Soil 39, 205-207. Doi: 10.1007/BF00018060

Belko, N., M. Zaman-Allah, N. Cisse, N.N. Diop, G. Zombre, J.D. Ehlers y V. Vadez. 2012. Lower soil moisture threshold for transpiration decline under water deficit correlates with lower canopy conductance and higher transpiration efficiency in droughttolerant cowpea. Funct. Plant Biol. 39(4), 306-322. Doi: http://dx.doi.org/10.1071/FP11282 
Beltrano, J., M.G. Ronco y M.C. Arango. 2006. Soil drying and rewatering applied at three grain developmental stages affect differentially growth and grain protein deposition in wheat (Triticum aestivum L.). Braz. J. Plant Physiol. 18(2), 341-350. Doi: 10.1590/S1677-04202006000200011

Blum, A. 2009. Effective use of water (EUW) and not water-use efficiency (WUE) is the target of crop yield improvement under drought stress. Field Crop. Res. 112, 119-123. Doi: 10.1016/j. fcr.2009.03.009

Bradford, M.M. 1976. A rapid and sensitive method for the quantitation of microgram quantities of protein utilizing the principle of protein-dye binding. Anal. Biochem. 72, 248-254. Doi: 10.1016/00032697(76)90527-3

Brodribb, T. 1996. Dynamics of changing intercellular $\mathrm{CO}_{2}$ concentration (ci) during drought and determination of minimum functional ci. Plant Physiol. 111, 179-185.

Cabrera, H.M. 2002. Respuestas ecofisiológicas de plantas en ecosistemas de zonas con clima mediterráneo y ambientes de alta montaña. Rev. Chil. Hist. Nat. 75, 625-637.

Cakmak, I. y W.J. Horst. 1991. Effect of aluminium on lipid peroxidation, superoxide dismutase, catalase, and peroxidase activities in root tips of soybean (Glycine max). Physiol. Plant. 83, 463-468. Doi: 10.1111/j.1399-3054.1991.tb00121.x

Cardona-Ayala, C.E., A.J. Jarma-Orozco, H. Araméndiz-Tatis, M. Perneth-Montaño y C.A. VergaraCórdoba. 2013. Gas exchange and mass distribution of the cowpea (Vigna unguiculata [L.] Walp.) under water deficit. Agron. Colomb. 31(3), 288296.

Cardona-Ayala, C.E., A.J. Jarma-Orozco y H. Araméndiz-Tatis. 2013. Mecanismos de adaptación a sequía en fríjol caupí (Vigna unguiculata [L.] Walp.). Rev. Colomb. Cienc. Hortic. 7(2), 277-288.

Claeys, H. y D. Inzé. 2013. The agony of choice: how plants balance growth and survival under waterlimiting conditions. Plant Physiol. 162, 1768-1779. Doi: http://dx.doi.org/10.1104/pp.113.220921

Costa, R.C.L., A.K.S. Lobato, C.F. Oliveira Neto, P.SP. Maia, G.A.R Alves y H.D. Laughinghouse. 2008. Biochemical and physiological responses in two Vigna unguiculata (L.) Walp. cultivars under water stress. J. Agron. 7(1), 98-101. Doi: 10.3923/ ja.2008.98.101

Fernandes-Santos, C., G.P. Pereira Lima y L.B. Morgado. 2010. Tolerância e caracterização bioquímica em feijão-caupi submetido a estressehídrico na pré-floração. Naturalia 33, 34-44.

Fisher, R.A. y R. Maurer. 1978. Drought resistance in spring wheat cultivars. Aust. J. Agric. Res. 29, 897-912. Doi: 10.1071/AR9791001

Forsythe, W. 1985. Física de suelos: manual de laboratorio. IICA, San José, Costa Rica.

Grassi, G. y F. Magnani. 2005. Stomatal, mesophyll conductance and biochemical limitations to photosynthesis as affected by drought and leaf ontogeny in ash and oak trees. Plant Cell Environ. 28, 834-849. Doi: 10.1111/j.1365-3040.2005.01333.x

Havir, E.A. y N.A. McHale. 1987. Biochemical and developmental characterization of multiple forms of catalase in tobacco leaves. Plant Physiol. 84, 450-455. Doi: 10.1104/pp.84.2.450

Ishiyaku, M.F. y H. Aliyu. 2013. Field evaluation of cowpea genotypes for drought tolerance and striga resistance in the dry savanna of the North-West Nigeria. Int. J. Plant Breed. Genet. 7(1), 47-56. Doi: 10.3923/ijpbg.2013.47.56

Iturbe-Ormaetxe, I., P.R. Escuredo, C. Arrese-Igor y M. Becana. 1998. Oxidative damage in pea plants exposed to water deficit or paraquat. Plant Physiol. 116, 173-181. Doi: 10.1104/pp.116.1.173

Lawlor, D.W. 2002. Limitation to photosynthesis in water-stressed leaves: stomata vs. metabolism and the role of ATP. Ann. Bot. 89, 871-885.

Lichtenthaler, H.K. 1987. Chlorophylls and carotenoids: Pigments of photosynthetic biomembranes. Method. Enzymol. 148, 350-382.

Martin, B. y N.A. Ruiz-Torres. 1992. Effects of waterdeficit stress on photosynthesis, its components and component limitations, and on water use efficiency in wheat (Triticum aestivum L.). Plant Physiol. 100, 733-739. Doi: 10.1104/pp.100.2.733

Nakano, Y. y K. Asada. 1981. Hydrogen peroxide is scavenged by ascorbate-specific peroxidase in spinach choloroplasts. Plant Cell Physiol. 22, 867-880.

Pompelli, M., R. Barata; H. Vitorino, E. Gonçalves, E. Rolim, M. Santos, J. Almeida, V. Ferreira, E. Lemos y L. Endres. 2010. Photosynthesis, photoprotection and antioxidant activity of purging nut under drought deficit and recovery. Biomass Bioener. 34(8), 1207-1215. Doi: 10.1016/j.biombioe.2010.03.011

Pungulani, L.L.M., J.P. Millner, W.M. Williams y M. Banda. 2013. Improvement of leaf wilting scoring in cowpea (Vigna sinensis (L.) Walp.): From qua- 
litative scale to quantitative index. Aust. J. Crop Sci. 7(9), 1262-1269.

Rosales-Serna, R., P. Ramírez-Vallejo, J.A. Acosta-Gallegos, F. Castillo-González y J.D. Kelly. 2000. Grain yield and drought tolerance of common bean under field conditions. Agrociencia 34(2), 153-165.

Singh, S.K. y K.R. Reddy. 2011. Regulation of photosynthesis, fluorescence, stomatal conductance and water-use efficiency of cowpea (Vigna unguiculata (L.) Walp.) under drought. J. Photochem. Photobiol B: Biol.105, 40-50. Doi: 10.1016/j.jphotobiol.2011.07.001

Taiz, L. y E. Zeiger. 2010. Plant physiology. $5^{\text {th }}$ ed. Sinauer Associates, Redwood City, CA. pp. 591-623.

Santos, C.F., G.P.P. Lima y L.B. Morgado. 2010. Tolerância e caracterização bioquímica em feijão-caupi submetido a estresse hídrico na pré-floração. Naturalia 33, 34-44.
Smirnoff, N. 1993. The role of active oxygen in the response of plants to water deficit and desiccation (Tansley Review No. 52). New Phytol. 125, 27-58. Doi: 10.1111/j.1469-8137.1993.tb03863.x

Tausz, M., H.S. Ircelj y D. Grill. 2004. The glutathione system as a stress marker in plant ecophysiology: is a stress-response concept valid? J. Exp. Bot. 55 (404), 1955-1962. Doi: 10.1093/jxb/erh194

Tripathy, B.C. y R. Oelmüller. 2012. Reactive oxygen species generation and signaling in plants. Plant Signal. Behavior 7(12), 1621-1633. Doi: 10.4161/ psb. 22455

Witta, S., L. Galiciab, J. Liseca, J. Cairnsc, A. Tiessend, J.L. Arause, N. Palacios-Rojasb y A.R. Fernie. 2012. Metabolic and phenotypic responses of greenhouse-grown maize hybrids to experimentally controlled drought stress. Mol. Plant 5(2), 401-417. Doi: $10.1093 / \mathrm{mp} / \mathrm{ssr} 102$ 